\title{
Capacidade funcional de indivíduos com insuficiência cardíaca avaliada pelo teste de esforço cardiopulmonar e classificação da New York Heart Association
}

\author{
Functional capacity of heart failure patients evaluated by \\ cardiopulmonary exercise test and New York Heart Association class \\ Danielle Aparecida Gomes Pereira', Roseane Santo Rodrigues², Giane Amorim Ribeiro Samora³, \\ Susan Martins Lage ${ }^{2}$, Maria Clara Nomam Alencar ${ }^{4}$, Verônica Franco Parreira', Raquel Rodrigues Britto ${ }^{1}$
}

RESUMO I O objetivo deste estudo foi avaliar a capacidade discriminativa da classificação da New York Heart Association (NYHA) em indivíduos com insuficiência cardíaca (IC) classes II e III por meio de variáveis do teste de esforço cardiopulmonar (TECP). Participaram do estudo 17 pessoas com IC classe

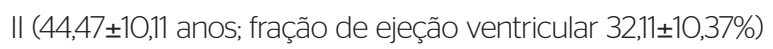

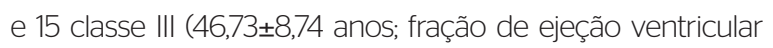
30,46 $\pm 10,23 \%$ ) da NYHA que realizaram TECP máximo em esteira ergométrica. De acordo com a distribuição dos dados, houve correlação de Pearson ou Spearman e, para comparação entre classes, utilizou-se o teste $t$ de Student Foram encontradas correlações significativas $(p<0,05)$ entre consumo de oxigênio $\left(\mathrm{VO}_{2}\right)$ e pulso de oxigênio $(r=0,76)$, idade $(r=0,35)$ e índice de massa corpórea $(r=0,45)$. Não houve correlação significativa entre fração de ejeção ventricular e as demais variáveis estudadas. Comparando as classes da NYHA, foram encontradas diferenças para $\mathrm{VO}_{2}$ e pulso de oxigênio $(p<0,05)$. Nesta pesquisa, as correlações refletem a complexidade de se analisar e entender o processo de disfunção da IC, considerando que provavelmente há outros fatores influenciando cada uma das variáveis estudadas. A classificação da NYHA foi efetiva na diferenciação dos indivíduos nos diferentes grupos, reforçando sua utilidade na caracterização de grupos de indivíduos com IC para fins de pesquisas científicas, assim como para avaliação de terapêuticas.

Descritores I teste de esforço; insuficiência cardíaca; classificação.

\begin{abstract}
IThe purpose of this study was to evaluate the capacity of the New York Heart Association (NYHA) classification to discriminate heart failure (HF) individuals classes II and III using cardiopulmonary exercise test (CPET) variables. Seventeen patients class II (age 44.47 \pm 10.11 years; ejection fraction $32.11 \pm 10.37 \%$ ) and 15 class III (age $46.73 \pm 8.74$ years; ejection fraction $30.46 \pm 10.23 \%$ ) of $\mathrm{NYHA}$ participated in this study. They did a maximal CPET in a treadmill ergometer. According to the distribution of data, correlation of Pearson or Spearman was performed and, to compare the functional classes of NYHA, the Student's $t$-test was used. Significant correlations $(p<0.05)$ between oxygen consumption $\left(\mathrm{VO}_{2}\right)$ and oxygen pulse $(r=0.76)$, age $(r=0.35)$ and body mass index $(r=0.45)$ were found. There was no significant correlation between ventricular ejection fraction and the others studied variables. Comparing the functional classes of NYHA, significantly differences were found to $\mathrm{VO}_{2}$ and oxygen pulse $(\mathrm{p}<0.05)$. In this study, the correlations reflect the complexity in analyzing and understanding the process of disfunction in HF, once probably other factors influence each of the variables studied. The classification of NYHA was effective to differentiate the patients with HF, reinforcing the indication of this stratification to identify different groups of HF patients to scientific researches and therapeutic interventions.
\end{abstract}

Keywords I exercise test; heart failure; classification.

Estudo desenvolvido no Laboratório de Avaliação e Pesquisa em Desempenho Cardiorrespiratório do Departamento de Fisioterapia junto ao Programa de Pós-Graduação em Ciências da Reabilitação da Universidade Federal de Minas Gerais (UFMG) - Belo Horizonte (MG), Brasil.

'Professora Doutora do Departamento de Fisioterapia da UFMG - Belo Horizonte (MG), Brasil.

Mestranda em Ciências da Reabilitação pela UFMG - Belo Horizonte (MG), Brasil.

${ }^{3}$ Doutoranda em Ciências da Reabilitação pela UFMG - Belo Horizonte (MG), Brasil.

${ }^{4}$ Médica Cardiologista do Hospital das Clínicas da UFMG - Belo Horizonte (MG), Brasil, e do Hospital Socor - Belo Horizonte (MG), Brasil.

Endereço para correspondência: Raquel Rodrigues Britto - Avenida Antônio Carlos, 6.627 - Pampulha - CEP: 31270-901 - Belo Horizonte (MG), Brasil - E-mail: rbrito@ufmg.br Apresentação: mai. 2011 - Aceito para publicação: dez. 2011 - Fonte de financiamento: Conselho Nacional de Pesquisa (CNPq), Coordenação de Aperfeiçoamento de Pessoal de Nível Superior (CAPES) e Fundação de Amparo à Pesquisa de Minas Gerais (FAPEMIG) - Conflito de interesses: nada a declarar - Parecer de aprovação do Comitê de Ética em Pesquisa da UFMG: ETIC 489/06 - Ad O1/07 


\section{INTRODUÇÃO}

A insuficiência cardíaca (IC) é uma síndrome clínica caracterizada por disfunção cardíaca que ocasiona suprimento sanguíneo inadequado para atender às necessidades metabólicas tissulares ${ }^{1}$. Ela é uma condição clínica endêmica em todo o mundo, de alto custo, incapacitante e com mortalidade elevada, representando grave problema de saúde pública ${ }^{1,2}$.

Tendo sido classicamente categorizada com base na intensidade de sintomas observados no exame clínico e de acordo com a sintomatologia apresentada durante o esforço ${ }^{1}$, tem como sintomas principais a fadiga e dispneia ${ }^{3}$, que ocasionam diminuição da capacidade funcional, dificultando a execução das atividades diárias e reduzindo a qualidade de vida ${ }^{3,4}$.

A classificação funcional da Nerw York Heart Association (NYHA) foi originalmente descrita em 1928 e recentemente atualizada (1994), sendo um instrumento de classificação com validade e confiabilidade estabelecidas ${ }^{5}$. A NYHA avalia o efeito sintomático da doença cardíaca, permitindo estratificar o grau de limitação imposto por ela para atividades cotidianas ${ }^{1,5}$. Segundo a associação, os indivíduos com IC são classificados em quatro: classe I - ausência de sintomas durante atividades cotidianas, com limitação para esforços semelhante à esperada em indivíduos saudáveis; classe II - sintomas desencadeados por atividades cotidianas; classe III - sintomas desencadeados em atividades menos intensas que as cotidianas; classe IV - sintomas em repouso ${ }^{1}$.

Além do valor prognóstico da classe funcional caracterizada em uma avaliação inicial, essa determinação é útil para avaliar qualidade de vida e resposta terapêutica e para determinar o melhor momento para intervenções ${ }^{1}$. Apesar disso, a determinação da classe funcional da NYHA é de caráter subjetivo e pode estar sujeita ao viés de diferentes interpretações.

$\mathrm{O}$ teste de esforço cardiopulmonar (TECP), por sua vez, é um procedimento não invasivo, válido e reprodutível, que objetiva avaliar a capacidade funcional $1^{6-8}$. É considerado padrão ouro para avaliação da capacidade aeróbica ou desempenho cardiorrespiratório e metabólico ${ }^{6,8}$, possibilitando avaliação global da resposta ao exercício e também informações relevantes para a tomada de decisão clínica.

O método de avaliação com gases expirados, característica básica do TECP, permite analisar de forma direta parâmetros ventilatórios, cardiovasculares e metabólicos, tais como: consumo de oxigênio $\left(\mathrm{VO}_{2}\right)$, produção de dióxido de carbono $\left(\mathrm{VCO}_{2}\right)$, frequência respiratória (f), volume corrente (VC) e frequência cardíaca $(\mathrm{FC})^{8}$. A partir desses parâmetros, é possível calcular outras variáveis referentes à capacidade aeróbica, entre elas: ventilação pulmonar (VE), equivalente ventilatório de oxigênio $\left(\mathrm{VE} / \mathrm{VO}_{2}\right)$, equivalente ventilatório de dióxido de carbono $\left(\mathrm{VE} / \mathrm{VCO}_{2}\right)$, razão de troca respiratória $\left(\mathrm{RER}=\mathrm{VCO}_{2} / \mathrm{VO}_{2}\right)$, fração expirada de oxigênio $\left(\% \mathrm{FEO}_{2}\right)$, fração expirada de dióxido de carbônico $\left(\% \mathrm{FECO}_{2}\right)$ e pulso de oxigênio $\left(\mathrm{PO}_{2}=\mathrm{VO}_{2} / \mathrm{FC}\right)^{9}$.

A partir do TECP, outra classificação ainda vigente diz respeito à identificação da importância do $\mathrm{VO}_{\text {2pico }}$ na predição de mortalidade de indivíduos com $\mathrm{IC}^{10}$. De acordo com o $\mathrm{VO}_{2 \text { pico }}$ atingido durante o exercício, quatro classes foram originalmente definidas - classe A: $>20 \mathrm{~mL} / \mathrm{min} / \mathrm{kg}$; classe B: $16-20 \mathrm{~mL} / \mathrm{min} / \mathrm{kg}$; classe C: $10-15 \mathrm{~mL} / \mathrm{min} / \mathrm{kg}$; classe D: $<10 \mathrm{~mL} / \mathrm{min} / \mathrm{kg}^{10}$.

Apesar dos mecanismos fisiopatológicos e suas relações envolvidas na limitação da capacidade funcional em indivíduos com IC não estarem totalmente esclarecidos, a intolerância ao exercício tem sido bem estabelecida nessa população pelo TECP e, clinicamente, por meio da classificação da NYHA. Dessa forma, o objetivo do estudo foi avaliar a capacidade discriminativa da associação em indivíduos com IC classes II e III por meio de variáveis do TECP.

\section{METODOLOGIA}

Trata-se de um estudo observacional do tipo transversal. Os participantes foram selecionados em serviços ambulatoriais de cardiologia da região metropolitana, após liberação médica. Foram incluídos indivíduos com IC, estágio $\mathrm{C}^{11}$, classes II e III da $\mathrm{NYHA}^{1}$ (titulados por um único avaliador), independente de sexo e etnia, que não praticassem atividade física regular, com idade entre 25 e 59 anos, estabilidade clínica há pelo menos dois meses ${ }^{12}$, fração de ejeção do ventrículo esquerdo (FEVE) em repouso $\leq 45 \%$ e que não apresentassem disfunção ortopédica ou neurológica que limitasse a realização de deambulação, história de doença pulmonar, angina instável, arritmias não controladas ou doença arterial obstrutiva periférica ${ }^{13}$.

As pessoas estudadas foram orientadas a manter medicação usual, fazer jejum de duas horas e evitar cafeína, cigarro e exercício físico no dia do teste ${ }^{14}$. Após a leitura e assinatura do Termo de Consentimento Livre e Esclarecido (TCLE), foi realizada entrevista para coleta 
de dados clínicos e sociodemográficos e mensuradas a massa corporal e altura.

O TECP (CPX Ultima, Medical Graphics ${ }^{\circledR}$, Miami, FL, USA) aconteceu em esteira ergométrica (Millenium Classic CI Inbramed/Inbrasport ${ }^{\circledR}$, Porto Alegre, RS) utilizando protocolo em rampa ${ }^{15-18}$ e compreendeu três fases: preparatória, incremental e recuperação. Durante o teste, realizado na presença de cardiologista, houve monitorização contínua do eletrocardiograma (Welch Allyn, Skaneateles Falls, NY, EUA) e da saturação periférica de oxigênio (Model 300 Series MEDIAD INC., Califórnia, USA). Após três minutos de repouso de pé na esteira, a cada dois minutos do teste e na fase de recuperação foram avaliadas pressão arterial (PA) e percepção subjetiva da intensidade do esforço pela escala modificada de Borg $^{18}$. O teste foi finalizado quando quociente respiratório $(\mathrm{QR})>1,1$ foi alcançado ou se observado algum dos critérios de interrupção de esforço máximo definidos pela diretriz do American College Cardiology/American Heart Association ${ }^{19}$. A temperatura ambiente permaneceu em $20 \pm 2^{\circ} \mathrm{C}$ e a umidade relativa do ar entre 50 e $70 \%{ }^{15}$.

As variáveis utilizadas para a análise foram $\mathrm{VO}_{2}$, $\mathrm{VE} / \mathrm{VCO}_{2}, \mathrm{PO}_{2}$, idade, índice de massa corpórea (IMC) e FEVE. Os dados estão apresentados como medidas de tendência central e dispersão. A distribuição dles foi analisada pelo teste de Shapiro-Wilk. Houve correlação de variáveis por meio do coeficiente de Pearson ou do coeficiente de Spearman. A magnitude das correlações realizadas nesse estudo foi classificada como fraca $(<0,50)$, moderada $(0,50-0,75)$ e alta $(>0,75)$. Para a comparação de variáveis entre as classes funcionais II e III da NYHA, utilizou-se o teste $t$ de Student. Em todos os testes estatísticos foram considerados significativos resultados com $\mathrm{p}<0,05$. Para análise dos dados foi utilizado o software SPSS ${ }^{\circledR}$ (Chicago, IL, USA), versão 13.0.

\section{RESULTADOS}

A amostra inicial foi composta por 44 voluntários. Doze foram excluídos devido ao tempo de teste inferior a seis minutos e/ou por apresentarem taquicardia ventricular e supraventricular durante o período de aquecimento anterior ao início do teste. Dessa forma, a amostra final foi composta por 32 indivíduos com IC classes II ( $n=17)$ e III ( $\mathrm{n}=15)$ da NYHA (Tabela 1$)$.

Em relação aos parâmetros metabólicos e ventilatórios analisados, foram encontradas, em geral, correlações de fraca à moderada magnitude (Tabela 2).
Tabela 1. Características dos participantes do estudo

$\begin{array}{lcc} & \begin{array}{c}\text { NYHA II } \\ (n=17)\end{array} & \begin{array}{c}\text { NYHA III } \\ (n=15)\end{array} \\ \text { Sexo (homens/mulheres)* } & 14 / 3 & 8 / 7 \\ \text { Idade (anos)** } & 44,47 \pm 10,11 & 46,73 \pm 8,75 \\ \text { IMC** } & 24,22 \pm 2,90 & 25,37 \pm 2,81 \\ \text { FEVE (\%)* } & 32,12 \pm 10,37 & 30,47 \pm 10,24 \\ \text { Etiologia da IC (\%) } & & \\ \text { Isquêmica } & 23,53 & 20,00 \\ \text { Chagas } & 17,65 & 6,66 \\ \text { Idiopática } & 29,41 & 46,66 \\ \text { Outras } & 29,41 & 26,68\end{array}$

NYHA: New York Heart Association; IMC: índice de massa corpórea; FEVE: fração de ejeção ventricular; IC: insuficiência cardíaca

*Valores expressos em frequência (valor n ou \%); **Valores expressos em média \pm desvio-padrão

Tabela 2. Correlações entre variáveis clínicas e obtidas pelo teste de esforço cardiopulmonar

$\begin{array}{lccc}\text { Variáveis } & \mathrm{r} & \mathrm{r}^{2} & \mathrm{p} \\ \mathrm{PO}_{2} \times \mathrm{VO}_{2} & 0,76 & (\mathrm{O}, 58) & <0,001 \\ \text { IdadexVO }_{2} & -0,35 & (\mathrm{O}, 12) & 0,048 \\ \mathrm{IMCXVE} / \mathrm{VCO}_{2} & 0,45 & (\mathrm{O}, 2 \mathrm{O}) & 0,009 \\ \mathrm{IMCXVO} & -0,45 & (\mathrm{O}, 20) & 0,010\end{array}$

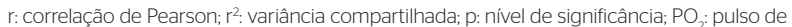
oxigênio em mL/kg/min.bpm'; $\mathrm{VO}_{2}$ : consumo de oxigênio em $\mathrm{mL} / \mathrm{kg} \cdot \mathrm{min}^{-1}$; IMC: índice de massa corpórea em kg/m²; VE/VCO ${ }_{2}$ : equivalente ventilatório para dióxido de carbono

Tabela 3. Comparação entre as classes II e III da New York Heart Association e o consumo de oxigênio $\left(\mathrm{VO}_{2 \text { pico }}\right)$

$\begin{array}{lcc}\text { Classes da NYHA } & \mathrm{VO}_{2 \text { pico }}\left(\mathrm{mL} / \mathrm{kg}_{\mathrm{min}} \mathrm{m}^{-1}\right) & \mathrm{p} \\ \text { NYHA II } & 27,27 \pm 5,80 & 0,001 \\ \text { NYHA III } & 18,19 \pm 3,40 & 0,001\end{array}$

NYHA: New York Heart Association; $(p<0,05)$

Os valores são expressos em média土desvio-padrão

Entre as variáveis $\mathrm{FEVE}, \%$ e $\mathrm{VO}_{2}, \mathrm{~mL} / \mathrm{kg} \cdot \mathrm{min}^{-1}$ $(\mathrm{r}=0,02 \mathrm{p}=0,92)$; FEVE, \% e $\mathrm{PO}_{2}, \mathrm{~mL} / \mathrm{min} \cdot \mathrm{bpm}^{-1}$ $(\mathrm{r}=0,01 ; \mathrm{p}=0,99)$; e entre a FEVE, \% e VE/ $\mathrm{VCO}_{2}$ $(r=0,03 \mathrm{p}=0,88)$ não houve correlação estatisticamente significativa.

Foram encontradas diferenças significativas entre os grupos da NYHA para $\mathrm{VO}_{2}$ (Tabela 3 ) e também entre as classes da NYHA e $\mathrm{PO}_{2}$ (NYHA II=12,42 $\pm 1,86 \mathrm{~mL} / \mathrm{min} \cdot \mathrm{bpm}^{-1}$ versus $\mathrm{NYHA}$ III $\left.=9,49 \pm 1,84 \mathrm{~mL} / \mathrm{min} \cdot \mathrm{bpm}^{-1} ; \mathrm{p}<0,01\right)$.

\section{DISCUSSÃO}

A identificação de correlações de fraca a moderada magnitude entre parâmetros metabólicos e ventilatórios do TECP, e ausência de correlação desses com a FEVE, representa a complexidade de se analisar e entender o processo de disfunção que ocorre na IC. Provavelmente há diferentes fatores influenciando cada uma das 
variáveis estudadas. No entanto, a principal contribuição desta pesquisa é indicar a capacidade discriminativa da classificação da NYHA quando avaliada pelo $\mathrm{VO}_{2} \mathrm{e}$ pelo $\mathrm{PO}_{2}$ medidos por meio do TECP.

No que diz respeito às variáveis $\mathrm{VO}_{2}$ e $\mathrm{PO}_{2}$, sabe-se que representam uma medida tradicional da capacidade aeróbica com valor prognóstico clínico e de mortalidade para a IC bem definidos ${ }^{20,21}$. Considerando que o déficit cronotrópico ao esforço observado em indivíduos com IC beta-bloqueados é variáve ${ }^{22}$, a alta correlação encontrada entre essas variáveis confirma que a maioria dos pacientes do presente estudo estava em doses otimizadas de beta-bloqueador.

Quando se considera a massa corporal do indivíduo (em kg) na unidade de medida do $\mathrm{PO}_{2}$, há um ganho no poder clínico da variável ${ }^{21}$. No presente estudo, observou-se maior correlação entre $\mathrm{VO}_{2}$ e $\mathrm{PO}_{2}$ quando se considerou a variável $\mathrm{PO}_{2} \mathrm{em} \mathrm{mL} / \mathrm{kg} / \mathrm{min} \cdot \mathrm{bpm}^{-1}$. Contudo, mesmo com a elevada correlação entre tais variáveis $(\mathrm{r}=0,76)$, quando se considera a variância compartilhada $\left(r^{2}=0,58\right)$ observa-se que somente $58 \%$ da variação do $\mathrm{VO}_{2}$ é explicada pelo $\mathrm{PO}_{2}$. Tal análise indica a multifatorialidade dessa relação, na qual outros fatores, além do $\mathrm{PO}_{2}$, têm influência na tolerância ao esforço nos indivíduos com IC avaliados.

Em estudo recente ${ }^{23}$, foi ressaltada a importância de se descrever o impacto do IMC nas variáveis fisiológicas obtidas do teste de esforço máximo, em vista da prevalência de obesidade na IC. Tal impacto não está bem definido na literatura, em se tratando de indivíduos com IC em uso de beta-bloqueador. Conforme dados da presente pesquisa, foi confirmada a correlação negativa de fraca magnitude entre IMC e $\mathrm{VO}_{2}(\mathrm{r}=-0,45)$, bem como a correlação positiva também de fraca magnitude entre $\mathrm{IMC}$ e VE/ $/ \mathrm{VCO}_{2}(\mathrm{r}=0,45)$. Além disso, no estudo ${ }^{23}$, foi encontrada correlação mais fraca entre $\mathrm{IMCxVE} / \mathrm{VCO}_{2}$ se comparada à $\mathrm{IMCxVO}_{2}$, diferentemente dos nossos resultados, em que não foram verificadas diferenças ao se comparar a magnitude dessas correlações. Contudo, pode-se inferir que a ausência de correlação entre IMC e as variáveis ergoespirométricas analisadas no presente estudo pode estar relacionada ao fato da amostra não incluir indivíduos obesos e apresentar baixa variabilidade em relação ao IMC.

Foi observada nesta pesquisa correlação negativa de fraca magnitude entre as variáveis $\mathrm{VO}_{2}$ e idade $(\mathrm{r}=-0,35)$, não havendo correlação significativa entre idade e $\mathrm{VE} / \mathrm{VCO}_{2} \mathrm{O}$ valor prognóstico do TECP foi avaliado em 1.605 indivíduos com $\mathrm{IC}^{24}$, dentre eles: jovens ( $\leq 45$ anos), adultos (46-65 anos) e idosos ( $\geq 66$ anos).
Foram encontradas correlações fracas entre idade e $\mathrm{VO}_{2 \text { pico }}(\mathrm{r}=-0,11 ; \mathrm{p}<0,01)$ e entre idade e $\mathrm{VE} / \mathrm{VCO}_{2}$ $(r=0,06 ; p<0,05)$, assim como o presente estudo. Esses dados confirmam que em indivíduos com IC, a capacidade funcional deixa de ter relação exclusivamente com a idade, levando em conta o impacto diferenciado da doença. Já em pessoas saudáveis, a relação entre idade e $\mathrm{VO}_{2}$ tem magnitude elevada, considerando a interferência direta na capacidade funcional ${ }^{25}$.

Pesquisa recente realizou Eco-Doppler, TECP, teste de caminhada de seis minutos e treinamento físico em 2.331 indivíduos com IC (FEVE $\leq 35 \%)^{26}$. Foram encontradas correlações fracas entre $\mathrm{FEVE} \mathrm{e} \mathrm{VO}_{\text {2pico }}$ $(\mathrm{r}=0,13 ; \mathrm{p}<0,01)$ e também entre FEVE e VE/ $\mathrm{VCO}_{2}$ $(r=-0,16 ; p<0,01)^{26}$. Apesar da disfunção ventricular esquerda poder alterar a capacidade de esforço, tem sido proposto que mecanismos compensatórios podem preservar a tolerância ao exercício, dentre eles: aumento do volume diastólico final, competência cronotrópica, aumento da extração de oxigênio, alteração da complacência ventricular esquerda ou, ainda, aumento do fluxo linfático pulmonar ${ }^{27}$. Assim, embora a intolerância ao exercício seja o maior sintoma destes pacientes, não parece existir relação direta entre a função sistólica e a capacidade de realização do exercício.

A partir da classificação de Weber ${ }^{10}$, podemos definir a amostra como de gravidade leve. Assim, além do que é reforçado pela literatura, a pequena variabilidade de valores alcançados de $\mathrm{VO}_{\text {2pico }}$ durante o TECP pode justificar a ausência de correlação entre função cardíaca e desempenho. Estudo anterior também aponta para uma grande variabilidade no teste de esforço de indivíduos com IC, quase sempre sem correlação com a $\mathrm{FEVE}^{27}$.

A classificação da NYHA, apesar de subjetiva, foi efetiva na diferenciação dos indivíduos com IC em relação à capacidade funcional medida por meio do $\mathrm{VO}_{2}$. Entretanto, a análise das variáveis do TECP tem relevância clínica por estimar com mais precisão a limitação funcional dos indivíduos com $\mathrm{IC}^{28} \mathrm{e}$, consequentemente, contribuir para o direcionamento do tratamento e das políticas de saúde para essa população.

Tendo em vista que os indivíduos avaliados pertenciam somente às classes II e III da NYHA e eram eutróficos, outros estudos, com cálculo amostral e que incluam sujeitos com IC de outras classes funcionais e com IMC de maior variabilidade, devem ser considerados em futuras investigações, de modo a avaliar a magnitude das relações envolvidas na intolerância ao esforço na IC. 


\section{CONCLUSÃO}

Nesse estudo, apesar de subjetiva, a classificação funcional de NYHA foi efetiva na diferenciação dos indivíduos com IC em relação à capacidade funcional medida por meio do $\mathrm{VO}_{2}$ e $\mathrm{PO}_{2}$. Esse achado reforça a indicação de tal estratificação no delineamento de pesquisas científicas e avaliação de intervenções terapêuticas. Além disso, a NYHA pode contribuir para a individualização de programas de reabilitação nessa população.

\section{REFERÊNCIAS}

1. Bocchi EA, Braga FGM, Ferreira SMA, Rohde LEP, Oliveira WA, Almeida DR, et al. III Diretriz Brasileira de Insuficiência Cardíaca Crônica. Arq Bras Cardiol. 2009;93(1):3-70.

2. Bocchi EA, Vilas-Boas F, Perrone S, Caamaño AG, Clausell N, Moreira MCV, et al. I Diretriz Latino-Americana para avaliação e conduta na insuficiência cardíaca descompensada. Arq Bras Cardiol. 2005;85(3):1-48.

3. Forgiarini LA Jr, Rubleski A, Douglas G, Tieppo J, Vercelino R, Dal Bosco $A$, et al. Evaluation of respiratory muscle strength and pulmonary function in heart failure patients. Arq Bras Cardiol. 2007;89(1):36-41.

4. Moraes RS, Nóbrega ACL, Castro RRT, Negrão CE, Stein R, Serra SM, et al. Sociedade Diretriz de Reabilitação Cardíaca. Arq Bras Cardiol. 2005;84(5):431-40.

5. Bennett JA, Riegel B, Bittner V, Nichols J. Validity and reliability of the NYHA classes for measuring research outcomes in patients with cardiac disease. Heart Lung. 2002;31(4):262-70.

6. Bader DS, Maguire TE, Balady GJ. Comparison of ramp versus step protocols for exercise testing in patients $>$ or $=60$ years of age. Am J Cardiol. 1999:83(1):11-4.

7. Yazbek Jr P, Tuda CR, Sabrag LMS, Zarzana AL, Batistella LR. Ergoespirometria: tipos de equipamentos, aspectos metodológicos e variáveis úteis. Rev Soc Cardiol Estado de São Paulo. 2001:11(3):682-94.

8. Arena R, Myers J, Guazzi M. The clinical importance of cardiopulmonary exercise testing and aerobic training in patients with heart failure. Rev Bras Fisioter. 2008;12(2):75-87.

9. Ferraz AS, Bocchi EA. Aplicações práticas da ergoespirometria na insuficiência cardíaca. Rev Soc Cardiol Estado de São Paulo. 2001;11(3):706-14.

10. Weber KT, Kinasewitz GT, Janicki JS, Fishman AP. Oxygen utilization and ventilation during exercise in patients with chronic cardiac failure. Circulation. 1982;65(6):1213-23.

11. Bonow RO, Bennett S, Casey DE Jr, Ganiats TG, Hlatky MA, Konstam MA, et al. ACC/AHA clinical performance measures for adults with chronic heart failure: a report of the American College of Cardiology/ American Heart Association Task Force on Performance Measures (Writing Committee to Develop Heart Failure Clinical Performance Measures) endorsed by the Heart Failure Society of America. J Am Coll Cardiol. 2005;46(6):1144-78

12. Kell R, Haunstetter A, Dengler TJ, Zugck C, Kübler W, Haass M. Do cytokines enable risk stratification to be improved in NYHA functional class III patients? Comparison with other potential predictors of prognosis. Eur Heart J. 2002:23(1):70-8

13. Gielen S, Adams V, Möbius-Winkler S, Linke A, Erbs S, Yu J, et al. Anti-inflammatory effects of exercise training in the skeletal muscle of patients with chronic heart failure. J Am Coll Cardiol. 2003:42(5): 861-8.

14. Deswal A, Petersen NJ, Feldman AM, Young JB, White BG, Mann DL. Cytokines and cytokine receptors in advanced heart failure: an analysis of the cytokine database from the Vesnarinone trial (VEST). Circulation. 2001;103(16):2055-9.

15. Guimarães Jl, Stein R, Vilas-Boas F. Normatização de técnicas e equipamentos para realização de exames em ergometria e ergoespirometria. Arq Bras Cardiol. 2003;80(4):457-64

16. Remme WJ, Swedberg K. Guidelines for the diagnosis and treatment of chronic heart failure. Eur Heart J. 2001;22(17):1527-60.

17. Silva OB, Sobral Filho DC. A new proposal to guide velocity and inclination in the ramp protocol for the treadmill ergometer. Arq Bras Cardiol. 2003;81(1):48-53.

18. Pereira DAG, Vieira DSR, Samora GAR, Lopes FL, Alencar MCN, Lage SM, et al. Reprodutibilidade da determinação do limiar anaeróbico em pacientes com insuficiência cardíaca. Arq Bras Cardiol. 2010;94(6):771-8.

19. Gibbons RJ, Balady GJ, Bricker JT, Chaitman BR, Fletcher GF, Froelicher $V F$, et al. ACC/AHA 2002 guideline update for exercise testing: summary article: a report of the American College of Cardiology/ American Heart Association Task Force on Practice Guidelines (Committee to Update the 1997 Exercise Testing Guidelines). Circulation. 2002;106(14):1883-92

20. Arena R, Myers J, Guazzi M. Cardiopulmonary exercise testing is a core assessment for patients with heart failure. Congest Heart Fail. 2011;17(3):115-9.

21. Oliveira RB, Myers J, Araújo CG, Arena R, Mandic S, Bensimhon D, et al. Does peak oxygen pulse complement peak oxygen uptake in risk stratifying patients with heart failure? Am J Cardiol. 2009;104(4):554-8.

22. Magrì D, Palermo P, Cauti FM, Contini M, Farina S, Cattadori G, et al. Chronotropic incompentence and functional capacity in chronic heart failure: no role of $\beta$-blockers and $\beta$-blocker dose. Cardiovasc Ther. 2012;30(2):100-8.

23. Horwich TB, Leifer ES, Brawner CA, Fitz-Gerald MB, Fonarow GC, HFACTION Investigators. The relationship between body mass index and cardiopulmonary exercise testing in chronic systolic heart failure. Am Heart J. 2009:158(4 Suppl):S31-6.

24. Arena R, Myers J, Abella J, Pinkstaff S, Brubaker P, Kitzman DW, et al. Cardiopulmonary exercise testing is equally prognostic in young, middle-aged and older individuals diagnosed with heart failure. Int J Cardiol. 2010;151(3):278-83.

25. Chan ED, Welsh $\mathrm{CH}$. Geriatric respiratory medicine. Chest. 1998;114(6):1704-33

26. Gardin JM, Leifer ES, Fleg JL, Whellan D, Kokkinos P, Leblanc MH, et al. Relationship of Doppler-Echocardiographic left ventricular diastolic function to exercise performance in systolic heart failure: the HFACTION study. Am Heart J. 2009:158(4):S45-52.

27. Benge W, Litchfield RL, Marcus ML. Exercise capacity in patients with severe left ventricular dysfunction. Circulation. 1980;61(5):955-9.

28. Costa RV, Junior AO, Serra SM, Nóbrega ACL. Respostas Ventilatórias e do Pulso de Oxigênio ao Exercício Dinâmico: Correlação com a massa muscular esquelética em postadores de insuficiência cardíaca crônica avaliados pela ergoespirometria. Rev SOCERJ. 2005;18(4):283-7. 